\title{
Predicting emission characteristics of volatile organic compounds from wet surface coatings
}

\author{
Sacide Alsoy Altinkaya* \\ Department of Chemical Engineering, Izmir Institute of Technology, Gulbahce Koyu, Urla-Izmir 35437, Turkey
}

\section{A R T I C L E I N F O}

\section{Article history:}

Received 18 March 2009

Received in revised form 29 July 2009

Accepted 30 July 2009

\section{Keywords:}

Mathematical model

VOC emission

Diffusion

Sorption isotherm

Evaporation

\begin{abstract}
A B S T R A C T
A mathematical model is developed to describe the emission characteristics of VOCs from homogeneous wet coating materials deposited on impermeable substrates. The model considers mass transfer in the material and in air, boundary layer resistance and the change in the coating thickness with time due to emission of VOC. Key features of the model are incorporation of concentration dependent VOC diffusion coefficients predicted from a physical model and nonlinear equilibrium isotherm at the coating/air interface. The model is applied to predict emission characteristics of MMA from acrylic based surface coatings. In an attempt to investigate the influences of equilibrium isotherm type and diffusion formalism on the predictions, simulations are performed with either constant or concentration dependent diffusivities and linear or nonlinear equilibrium isotherms. The lowest MMA concentration in air is predicted by incorporating the concentration dependent diffusivity and nonlinear equilibrium isotherm. The results suggest that assuming the diffusivity of MMA constant or equilibrium isotherm linear may lead to wrong conclusions about the emission rates from wet coatings. The model is general, fully predictive and can be used to predict emission rates of different VOCs from different coating materials if diffusion and thermodynamic parameters are available.
\end{abstract}

(c) 2009 Elsevier B.V. All rights reserved.

\section{Introduction}

Interior coating materials have been recognized as a major source of volatile organic compounds (VOCs) in buildings. VOCs can cause adverse effects on human health such as headache, tiredness, eye, nose, or throat irritations, dry cough and dizziness [1]. In order to control the VOC emission, thus, to prevent indoor air pollution problems, it is essential to study the emission characteristics. Currently, the characterization of VOC emission from coating materials is done either directly or indirectly. Although, direct measurements provide realistic results, they are usually costly, time-consuming and cannot provide insight into the mechanisms of the emission process. Indirect characterization of VOC emission is based on predictions from mathematical models.

Numerous models have been developed in the literature to describe VOC emissions from coating materials. These models can be categorized into two groups as empirical and physical models. The first- and second-order decay models [2-6] are all empirical in nature. In these models, the initial emission rate of the material and decay rate constants are determined experimentally. Even though the empirical models were used to correlate the emission data, they cannot provide information on the emission rates under different

\footnotetext{
* Tel.: +90 232750 6658; fax: +90 2327506645 .

E-mail address: sacidealsoy@iyte.edu.tr.
}

environmental conditions or for different coating materials. The physical models are developed based on the VOC-mass transfer principle. Two main mechanisms contribute to the VOC emission from the coating materials: the diffusion of VOC in the material as a result of concentration and/or temperature gradient and interfacial mass transfer due to evaporation of VOC from the surface as a result of interaction with air. In some of the earlier physical models, VOC emission was assumed to be governed by external mass transfer effects [7-10] and they were only successful in predicting the short-term emissions. Experimental studies have indicated that VOC emission is mainly controlled by internal diffusion especially at later stages of emission when material becomes dry. In a few diffusion models, external convective mass transfer resistance was neglected and only internal diffusion within the material was taken into account $[11,12]$. More complete models were developed by taking into account combined effects of internal and external mass transfer resistances [13-28]. In these models, the material is treated as a homogenous medium that can be characterized by an effective diffusion coefficient. Thus, the mass transfer through the material is described by Fick's second law. The initial concentration of VOC was assumed to be either constant [13-22,24,25,28] or nonuniform $[23,26,27]$. To predict the concentration of VOCs in air, macroscopic mass balances were generally used [16-21,23-28], on the other hand, in a few studies, two- or three-dimensional momentum and mass transfer equations were solved [13-15,22]. Most of these models assume that equilibrium relationship is linear and the 
diffusivity of VOC in the coating material is constant. Although these assumptions may be suitable to predict the emission rates from dry materials, they cannot be generalized and may cause obvious errors under certain conditions. In the case of wet coating materials, the initial concentration of VOC in the material is high, thus, the dependence of VOC diffusion coefficient on VOC concentration cannot be ignored and equilibrium relationship deviates from linearity. In a model developed by Yang et al. $[13,15,22]$ for simulating VOC emissions from wet coating materials, the diffusion was considered a thermally activated process and the temperature dependence of the diffusivity was described by Arhenius equation. To describe the concentration dependency, a third power empirical equation was used. The empirical nature of the equation and infinitely small diffusivity predictions at very low VOC concentrations limit the general applicability of the model. Haghighat and Huang [19] used a second-order empirical equation to simulate both the wet and dry phases of the wet materials. This diffusion model does not predict infinitely small diffusivities, however, the empirical factors in the model confine its relevancy. Both in the models of Yang et al. [13,15,22] and Haghighat and Huang [19], Henry's law was used as an equilibrium relationship although this thermodynamic expression is valid at low concentrations.

In this study, a comprehensive emission model has been developed to predict VOC emissions from wet coating materials. The model takes into account mass transfer in the material and in air, boundary layer resistance and the change in the coating thickness with time due to emission of VOC. Different from the models in the literature, a physically reliable expression to predict VOC diffusivity as a function of concentration and nonlinear equilibrium relationship were incorporated in the model. The model was applied to simulate methyl methacrylate emission from an acrylic coating in which methylmethacrylate (MMA) butylacrylate (BA) copolymer was used as a resin. Based upon model predictions, the applicability of linear equilibrium isotherm or constant diffusivity approximations was discussed. In addition, the influences of initial VOC concentration, coating thickness, air velocity, air temperature and air exchange rate on the emission rates were investigated and $8 \mathrm{~h}$ time averaged air concentrations were predicted.

\section{Theory}

The schematic drawing of a wet coating material applied to a substrate is shown in Fig. 1. The coating material is assumed to be homogeneous and has a uniform initial VOC concentration while the substrate is considered to be impermeable to VOC in the coating material. In addition, there is no chemical reaction between the coating material and VOC, mass transfer in the coating material is one dimensional, driven only by concentration difference and diffusion of VOC is Fickian type. When the coating material is exposed to air which is well mixed then, VOC will evaporate from the film surface and temperature gradient in the coating material due to evaporation is assumed to be negligible. To describe equilibrium

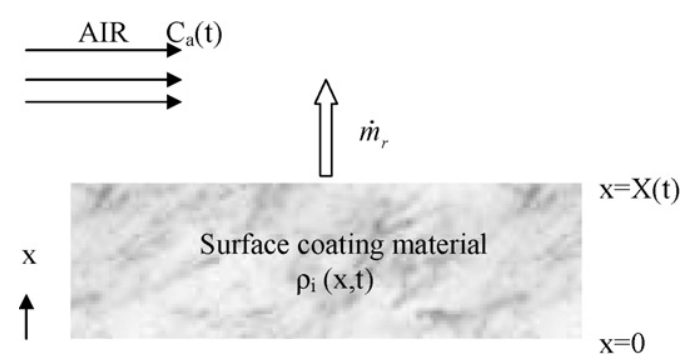

Fig. 1. Schematic representation of model geometry. at material-air interface, nonlinear sorption isotherm was adopted and the coating material was treated as a pseudobinary system.

Under these assumptions, the transient VOC-mass transfer in the coating material is described by the following equation:

$\frac{\partial \rho_{1}}{\partial t}=\frac{\partial}{\partial x}\left(D \frac{\partial \rho_{1}}{\partial x}\right)$

where $\rho_{1}$ and $D$ represent the concentration and diffusion coefficient of VOC in the coating. An expression for the possible decrease in the thickness of the coating material, $X$, due to evaporation is obtained by applying jump mass balance at the coating-air interface.

$\frac{d X}{d t}=-\left[\frac{-D \frac{\partial \rho_{1}}{\partial x} \hat{V}_{1}}{\left(1-\sum_{j=1}^{N-1} \rho_{j} \hat{V}_{j}\right)}\right]$

where $N=2$ for a binary system and the summation term in this case corresponds to volume fraction of the component 1 $\left(N=2 \rightarrow \sum_{j=1}^{N-1} \rho_{j} \hat{V}_{j}=\rho_{1} \hat{V}_{1}\right)$. Initial and boundary conditions required for the solution of Eqs. (1) and (2) are as follows:

$$
\begin{aligned}
& t=0 \quad \rho_{1}(0, x)=\rho_{10} \\
& x=0 \quad \frac{\partial \rho_{1}}{\partial x}=0 \\
& x=X(t) \quad-\left(D \frac{\partial \rho_{1}}{\partial x}\right)-\rho_{1} \frac{d X}{d t}=k_{1}^{G}\left(P_{1, \text { int }}^{G}-P_{1 b}^{G}\right)
\end{aligned}
$$

In Eqs. (2)-(5) $\hat{V}_{1}$ and $\rho_{10}$ correspond to specific volume and initial concentration of VOC in the coating while $k_{1}^{G}$ is the mass transfer coefficient of VOC in the gas phase and $P_{1 \text {,int }}^{G}$ and $P_{1 b}^{G}$ represent the partial pressure of VOC at the coating/air interface and in the bulk phase, respectively.

The change in VOC concentration in air, $C_{a}$, can be expressed as follows:

$V \frac{d C_{a}}{d t}=\dot{m}_{r}-Q C_{a}$

The rate of emission of VOC, $\dot{m}_{r}$, is defined by the following equation.

$\dot{m}_{r}=k_{1}^{G} A\left(P_{1, \text { int }}^{G}-P_{1 b}^{G}\right)$

and the relationship between the concentration of VOC in air, $C_{a}$, and $P_{1 b}^{G}$ is given by ideal gas law.

$P_{1 b}^{G}=C_{a} R T$

In Eqs. (6)-(8), $V, A$ and $Q$ denote the volume of the room, surface area of the coating and air exchange rate, respectively.

In two previous studies, the concentration dependence of VOC diffusivity was described by either second- or third-order polynomials $[13,19]$. Empiric nature of these expressions limits their use for different materials. In this study, a physical model based on Vrentas and Duda free volume theory was used [29,30]. According to this theory, the self-diffusion coefficient of the solvent, $D_{1}$, is expressed as follows:

$$
\begin{aligned}
D_{1}= & D_{o} \exp \left(-\frac{E}{R T}\right) \\
& \times \exp \left(-\frac{\omega_{1} \hat{V}_{1}^{*}+\omega_{2} \hat{V}_{2}^{* \xi}}{\omega_{1} \frac{K_{11}}{\gamma}\left(K_{21}-T_{g 1}+T\right)+\omega_{2} \frac{K_{12}}{\gamma}\left(K_{22}-T_{g 2}+T\right)}\right)
\end{aligned}
$$


In Eq. (9), $D_{0}, E, \omega_{i}$ denote the preexponential constant, activation energy and weight fraction of component $i$ (1:VOC 2:coating), respectively. The parameter groups $K_{11} / \gamma$ and $K_{21}-T_{g 1}$ are free volume parameters of the penetrant while the groups $K_{12} / \gamma$ and $K_{22}-T_{g 2}$ are free volume parameters of the polymer. In addition, $\hat{V}_{i}^{*}$ is the specific critical hole free volume of component $i$ and the parameter $\xi$ is defined as the ratio of the penetrant to polymer jumping units involved in a diffusive step. Duda et al. [31] proposed following relationship to couple the self-diffusion coefficients to mutual diffusion coefficient, $D$.

$D=D_{1} Q$

where thermodynamic factor $Q$ is defined as follows in terms of weight fractions, $\omega_{1}$ and $\omega_{2}$ and chemical potential $\left(\mu_{1}\right)$ gradient:

$Q=\frac{\omega_{1} \omega_{2}}{R T}\left(\frac{\partial \mu_{1}}{\partial \omega_{1}}\right)_{T, P}$

To describe thermodynamic equilibrium at the coating-air interface, Flory Huggins theory was used [32]. According to this theory, the activity of the VOC in vapor phase, $a_{1}$, is related to its volume fraction in the coating material, $\varphi_{1}$, by:

$\ln a_{1}=\ln \frac{P_{1, \text { int }}^{G}}{P_{1}^{s a t}}=\frac{\mu_{1}-\mu_{1}^{o}}{R T}=\ln \varphi_{1}+\varphi_{2}+\chi \varphi_{2}^{2}$

where $P_{1}^{\text {sat }}$ is the vapor pressure of VOC and $\chi$ represents Flory Huggins interaction parameter. At low concentrations, the Flory Huggins theory approaches to Henry's law, which relates the VOC concentration at the coating-air interface, $\rho_{1 \text {,int }}^{G}$, to that in air, $\rho_{1 b}^{G}$, linearly through a partition coefficient, $K$.

$\rho_{1, \text { int }}^{G}=K \rho_{1 b}^{G}$

An equation which describes the partition coefficient in terms of Flory Huggins interaction parameter is obtained by applying the limit that $\varphi_{1} \rightarrow 0$ [33].

$K=\frac{R T}{P_{1}^{s a t} M_{1} \hat{V}_{1} \exp (1+\chi)}$

where $M_{1}$ is the molecular weight of the VOC. The final form of the mutual diffusion coefficient depends on the thermodynamic theory used to define thermodynamic factor $Q$. If Flory Huggins theory is used, then $Q$ is obtained as follows by evaluating the chemical potential gradient from Eq. (12).

$Q=\varphi_{2}^{2}\left(1-2 \chi \varphi_{1}\right)$

In the case of Henry's law, the chemical potential expression and the thermodynamic factors are defined by Eqs. (16) and (17), respectively.

$\frac{\mu_{1}-\mu_{1}^{o}}{R T}=\ln \frac{\varphi_{1} R T}{M_{1} \hat{V}_{1} K P_{1}^{s a t}}$

$Q=\varphi_{2}$

The relationship between the concentration, $\rho_{1}$, and the volume fraction of the VOC, $\varphi_{1}$, is given by the following equation.

$\varphi_{1}=\rho_{1} \hat{V}_{1}$

\section{Numerical solution of the model equations and evaluation of model parameters}

Model equations shown in Eqs. (1)-(8) are nonlinear, coupled partial and ordinary differential equations. To solve these equations numerically, they were first discretized using the finite difference approximation with a variable grid size. To facilitate numerical treatment of the moving boundary, a coordinate transformation
Table 1

Input parameters used in the simulations.

\begin{tabular}{lc}
\hline Parameter & Value \\
\hline$K_{11} / \gamma\left(\mathrm{cm}^{3} / \mathrm{g} \mathrm{K}\right)$ & 0.00262 \\
$K_{12} / \gamma\left(\mathrm{cm}^{3} / \mathrm{g} \mathrm{K}\right)$ & 0.00013 \\
$K_{21}-T_{g 1}(\mathrm{~K})$ & -120.2 \\
$K_{22}-T_{\mathrm{g} 2}(\mathrm{~K})$ & -81.2 \\
$\hat{V}_{1}^{*}\left(\mathrm{~cm}^{3} / \mathrm{g}\right)$ & 0.871 \\
$\hat{V}_{2}^{*}\left(\mathrm{~cm}^{3} / \mathrm{g}\right)$ & 0.83 \\
$D_{0}^{*}\left(\mathrm{~cm}^{2} / \mathrm{s}\right)$ & $4.58 \times 10^{-6}$ \\
$\xi$ & 0.298 \\
$E(\mathrm{cal} / \mathrm{mol})$ & 0 \\
$\chi$ & 0.55 \\
$\hat{V}_{1}\left(\mathrm{~cm}^{3} / \mathrm{g}\right)$ & 1.067 \\
$\hat{V}_{2}\left(\mathrm{~cm}^{3} / \mathrm{g}\right)$ & 0.934 \\
$V\left(\mathrm{~m}^{3}\right)$ & 27 \\
$A\left(\mathrm{~m}^{2}\right)$ & 9 \\
$P_{1}^{\mathrm{sat}}(\mathrm{Pa})$ & \\
$23^{\circ} \mathrm{C}$ & 4417 \\
$27^{\circ} \mathrm{C}$ & 5468 \\
$30^{\circ} \mathrm{C}$ & 6387
\end{tabular}

was used, and, to reduce the stiffness of the equations, they were dimensionalized and updated according to the coordinate transformation [34]. Resulting algebraic equations were solved using an IMSL routine called DNEQNF.

In a previous study conducted in our group, diffusion coefficients and sorption isotherm of MMA in methyl methacrylate-butyl acrylate copolymer (MMA-BA) have been measured [35] and the data were correlated well with the Vrentas and Duda free volume theory and Flory Huggins theory, respectively. The free volume parameters and the Flory Huggins interaction parameter required in Eqs. (9)-(12) were obtained from this study and they are listed in Table 1 . The mass transfer coefficient of MMA in air was calculated from a correlation proposed by Haghighat and Zhang [36]. The saturated vapor pressure and the physical properties of MMA were obtained from Daubert and Danner [37] and they are also listed in Table 1.

\section{Results and discussion}

The emission characteristics of VOCs from the coatings are significantly influenced by the kinetic and thermodynamic parameters. Fig. 2 shows the equilibrium isotherms of MMA predicted from the Flory Huggins theory and Henry's law, respectively. At low concentrations, two theories produce similar results up to

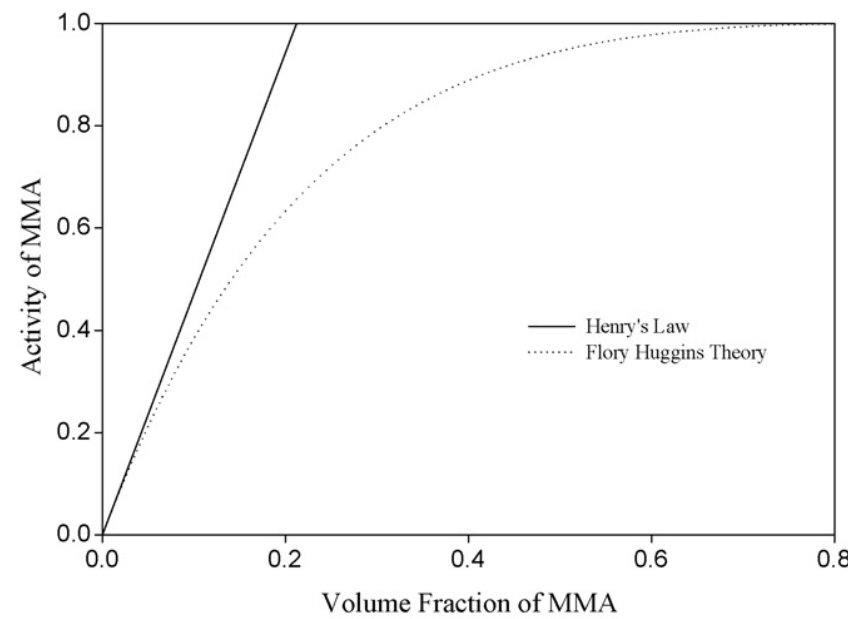

Fig. 2. The equilibrium isotherms of MMA in the acrylic coating predicted from Henry's law and Flory Huggins theory. 


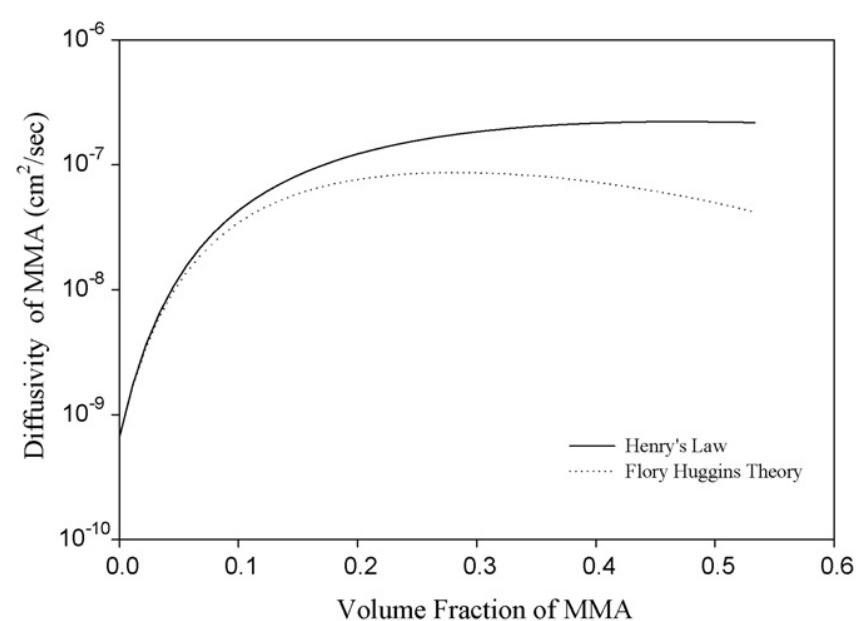

Fig. 3. The change of diffusivity of MMA in the acrylic coating with respect to its concentration.

a volume fraction of about 0.05 , on the other hand, significant deviations occur at higher volume fractions. Similar observation has been reported by Tiffonnet et al. [38]. They have measured that the sorption isotherms of acetone on chipboard and gypsum board are nonlinear. The literature on the sorption studies indicate that the choice of the equilibrium isotherm depends not only on the penetrant concentration but also on the glass transition temperature of the polymer. Henry's law can be used for low penetrant concentrations in rubbery polymers, on the other hand, for glassy polymers, the sorption isotherm becomes nonlinear even at relatively low penetrant concentrations [39-42]. These results certainly suggest that predicting VOC emission rates from wet coating materials where the initial VOC contents are high should be based upon a nonlinear equilibrium isotherm. The diffusivity of MMA was predicted by a combination of self-diffusion coefficient and thermodynamic factor and Fig. 3 illustrates that it is sensitive to the equilibrium relationship used for calculating the thermodynamic factor especially at high concentrations. It is noted that that as the volume fraction of MMA drops from 0.2 to 0.02 , the diffusivity of MMA decreases by a factor of 30 and 20 when the thermodynamic factor is predicted from Henry's law and the Flory Huggins theory, respectively. Such a drastic change in the diffusivity at room temperature can be attributed to the glass transition temperature of the coating $\left(32^{\circ} \mathrm{C}\right)$ close to room temperature. According to Vrentas Duda free volume theory used in this study, the concentration dependence of the mutual diffusion coefficient is greatest near $T_{g}$. For polymeric materials with glass transition temperature much lower than the room temperature, the concentration dependence of VOC diffusivity is usually negligible. This is due to fact that at room temperature, the material has sufficient free volume and the decrease in the VOC amount in the coating due to its desorption does not significantly change its diffusion rate. Based on these arguments, it can concluded that the concentration dependence of VOC diffusivity in polymeric surface coating materials should be taken into account when there is a big change in the concentration of VOC during total emission period, which is the case for wet coatings and when the glass transition temperature of the coating material is around room temperature and higher. In the literature Yang et al. $[13,15,22]$ and Haghighat and Huang [19] used second- and third-order empirical equations to describe concentration dependence of VOC diffusivities. Different from these approaches, the diffusion model used in this study has a physical background and can be applied to any VOC-coating system if its parameters are available. Part of the parameters in the diffusion theory $\left\{K_{11} / \gamma, K_{12} / \gamma, K_{21}-T_{g 1}, K_{22}-T_{g 2}, \hat{V}_{1}^{*}, \hat{V}_{2}^{*}\right\}$ can be determined from pure-component viscosity data as a function of temperature, or molar volumes of the pure components at $0 \mathrm{~K}$, and the others $\left\{D_{0}, E, \xi\right\}$ are regressed from the diffusivity data collected for the system of interest. The Flory Huggins interaction parameter, $\chi$, can be determined from equilibrium sorption isotherm using Eq. (12). The procedure for calculating these parameters is described in detail by Zielinski and Duda [43]. In addition to its physical basis, Vrentas Duda free volume theory is reliable and can be able to explain the observations in the literature. For example, Cox et al. [44] have reported that the emission rate of npentadecane from vinyl flooring is almost constant at temperatures below the glass transition temperature of the vinyl flooring $\left(49^{\circ} \mathrm{C}\right)$, however, increased by $430 \%$ as the temperature is increased from $35^{\circ} \mathrm{C}$ to $75^{\circ} \mathrm{C}$. According to Vrentas Duda free volume theory, at $T<T_{g}$ polymer chains do not have enough mobility to attain equilibrium configuration, thus, extra hole free volume becomes trapped within the polymer. As the polymer goes from glassy to rubbery state, the chain relaxation occurs at a faster rate and trapped hole free volume becomes available which significantly enhances the rate of migration of small molecules.

In order to investigate the influences of equilibrium isotherm type and diffusivity expression on the predictions of MMA concentration in air, simulations have been performed for four different cases as shown in Fig. 4a and b: In cases 1 and 2, the diffusivity is concentration dependent and the equilibrium isotherm is described by Flory Huggins theory (nonlinear) and Henry's law (linear), respectively while in cases 3 and 4, the diffusivity is considered constant and nonlinear and linear equilibrium isotherms are utilized, respectively. It is evident from Fig. 4a and b that the predictions from all cases overlap with each other at very low initial
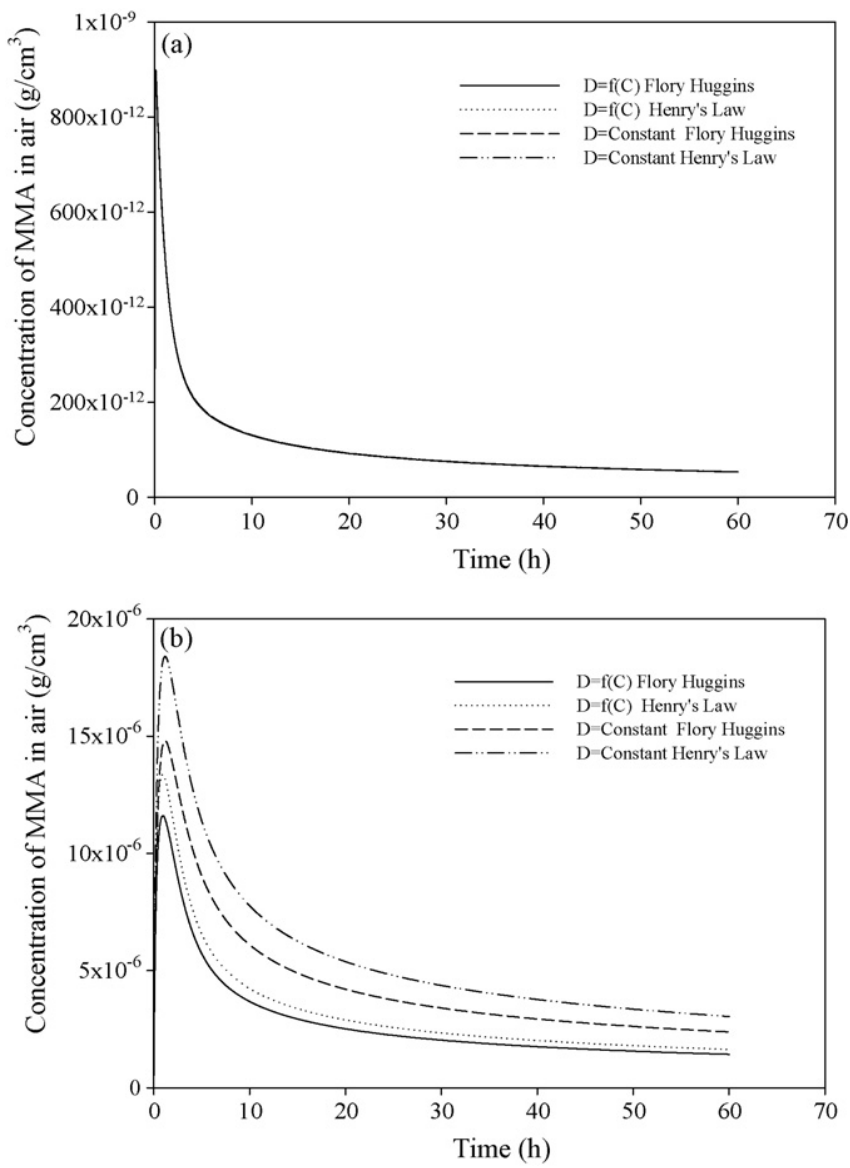

Fig. 4. The effects of diffusivity and equilibrium isotherm expressions on the predictions of MMA concentrations in air. 


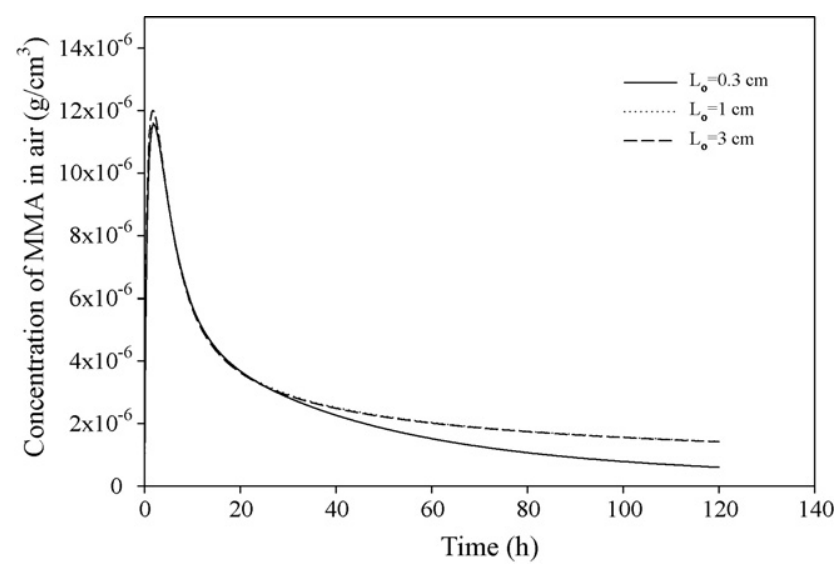

Fig. 5. The effect of the coating thickness on the concentration of MMA in air.

MMA content ( $100 \mathrm{ppm}$ ) which corresponds to the typical amount in dry materials, however, deviations occur at high concentrations $\left(0.2 \mathrm{~g} / \mathrm{cm}^{3}\right)$. Simulation results obtained for an initial MMA concentration of $0.2 \mathrm{~g} / \mathrm{cm}^{3}$ have shown that assuming diffusivity of MMA constant leads to predict higher MMA concentrations in air due to lower mass transfer resistance in the coating, thus, higher emission rates. In addition, the use of a linear rather than a nonlinear equilibrium isotherm raises MMA level in air since in this case the diffusivity of MMA and its equilibrium concentration at the coating surface are predicted higher. To examine the effects of environmental conditions on the emission of MMA from wet acrylic coating, a series of simulations have been conducted. In the simulations, all parameters are kept the same as those of the reference case except for the parameter investigated and the diffusivities and equilibrium surface concentrations are predicted from the Vrentas Duda free volume theory and Flory Huggins thermodynamic theory, respectively. The input data and the parameters used in the simulations are listed in Tables 1 and 2, respectively. Fig. 5 shows the influence of coating thickness on the MMA concentrations in air. At short times, the coating thickness has no influence on MMA levels in air while at longer periods, the MMA concentrations in air increase with the increased coating thickness due to presence of more MMA in the thicker coating. These predictions are in agreement with those reported by Yang et al. [15]. Their results indicate that emission rates from all wet coatings are approximately the same during the first hour after that the thicker coatings were found to have higher emission rates compared with the thinner coatings. The MMA concentration in air depends on its initial amount in the coating. Fig. 6 shows that increasing the initial MMA concentration from $0.1 \mathrm{~g} / \mathrm{cm}^{3}$ to $0.2 \mathrm{~g} / \mathrm{cm}^{3}$ raises maximum MMA level in the air by a factor of 4 . The predictions obtained by Yang et al.

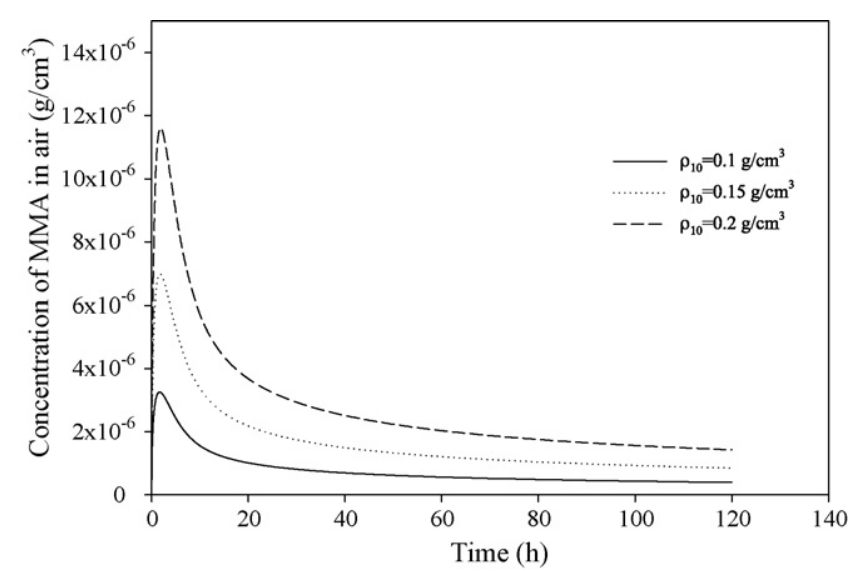

Fig. 6. The effect of initial concentration of MMA in the coating on its concentration in air.

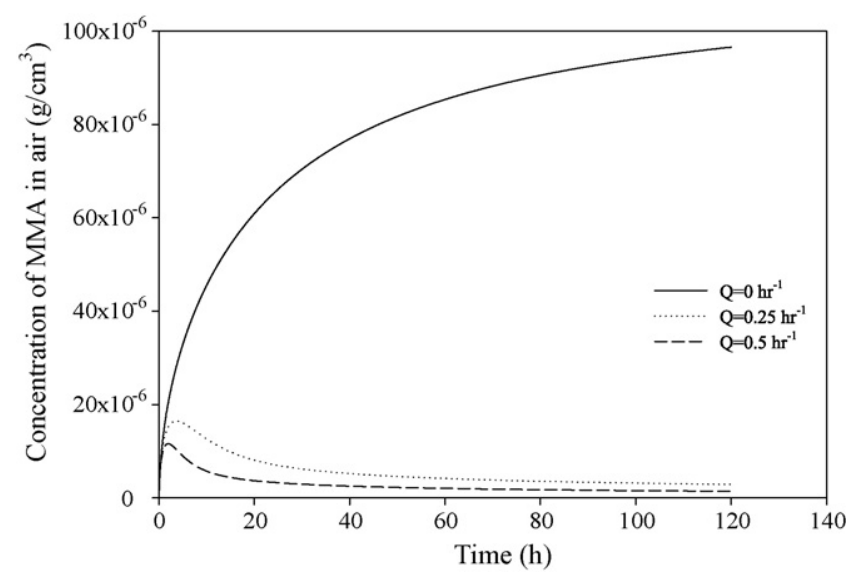

Fig. 7. The effect of air exchange rate on the concentration of MMA in air.

[14] also indicated that the VOC concentration in the chamber air depends linearly on the initial VOC content in the coating. To control the VOCs level in indoor air, the air exchange rate can be varied. Fig. 7 shows that the concentration of MMA in air increases very rapidly when the air exchange rate is zero, while a maximum in MMA concentration is followed by a decrease at high air exchange rates. Similar to the predictions of Cheng et al. [18], the MMA concentration in the bulk air decreases when air is exchanged more frequently. In addition to air exchange rates, the air velocities can also be changed with the ventilation strategy in the buildings. As opposed to the significant effect of the air exchange rate on the emission of MMA, the results in Fig. 8 show that the air velocity

Table 2

List of parameters changed in the simulations.

\begin{tabular}{|c|c|c|c|c|c|}
\hline Code of simulated case & Air exchange rate $\left(\mathrm{h}^{-1}\right)$ & Air velocity $(\mathrm{m} / \mathrm{s})$ & Air temperature $\left({ }^{\circ} \mathrm{C}\right)$ & Initial concentration of MMA $\left(\mathrm{g} / \mathrm{cm}^{3}\right)$ & Initial coating thickness $(\mathrm{cm})$ \\
\hline $\mathrm{C} 1$ & 0.5 & 0.1 & 23 & 0.2 & 1 \\
\hline $\mathrm{C} 2$ & 0.5 & 0.05 & 23 & 0.2 & 1 \\
\hline C3 & 0.5 & 0.5 & 23 & 0.2 & 1 \\
\hline C4 & 0.5 & 0.1 & 27 & 0.2 & 1 \\
\hline C5 & 0.5 & 0.1 & 30 & 0.2 & 1 \\
\hline C6 & 0.5 & 0.1 & 23 & 0.15 & 1 \\
\hline $\mathrm{C} 7$ & 0.5 & 0.1 & 23 & 0.1 & 1 \\
\hline C8 & 0.25 & 0.1 & 23 & 0.2 & 1 \\
\hline $\mathrm{C} 9$ & 0. & 0.1 & 23 & 0.2 & 1 \\
\hline C10 & 0.5 & 0.1 & 23 & 0.2 & 0.3 \\
\hline C11 & 0.5 & 0.1 & 23 & 0.2 & 3 \\
\hline C12 & 0.5 & 0.1 & 23 & $1 \times 10^{-4}$ & 1 \\
\hline
\end{tabular}




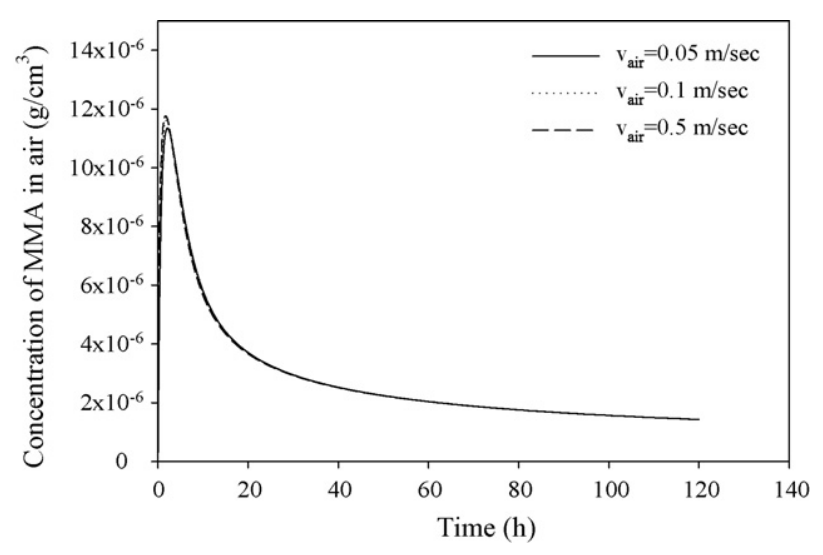

Fig. 8. The effect of air velocity on the concentration of MMA in air.

does not have any influence on the MMA accumulation in air. This is an indication of diffusion controlled emission of MMA even at the lowest air velocity investigated. Negligible effect of the air velocity on the emission rate of VOCs has also been reported by Yang et al. [15] for a similar velocity range used in this study. The influence of air temperature on the MMA levels in air is illustrated in Fig. 9. The results indicate that the increase in air temperature from $23^{\circ} \mathrm{C}$ to $30^{\circ} \mathrm{C}$ does not lead to significant difference in the predictions of MMA concentrations in air. These predictions are consistent with the conclusions of Yang et al. [15]. It can be noted from Figs. 5-9 that initially MMA accumulates very fast in air, however, at longer emission periods, MMA level in air changes slowly since the internal diffusion limitation increases with the lower diffusivity of MMA in the coating.

The effect of VOC levels inside the buildings on human health is usually evaluated in terms of $8 \mathrm{~h}$ exposure, therefore, 8 -h time weighted average concentrations, $\bar{C}_{a i r}$, were evaluated based on the following equation:

$\bar{C}_{\text {air }}=\frac{1}{8} \int_{t=0}^{t=8 \mathrm{~h}} C_{\text {air }}(t) d t$

It is evident from Fig. 10 that the average MMA concentrations predicted using different expressions for diffusion and equilibrium isotherms differ from each other and the difference in the predicted values becomes more significant with the increased initial MMA content in the coating. This result suggests that incorporating a nonlinear equilibrium isotherm and the concentration dependence of the MMA diffusivities are critical to predict reliable emission rates from the wet coating. Fig. 11 shows that 8 -h time weighted average

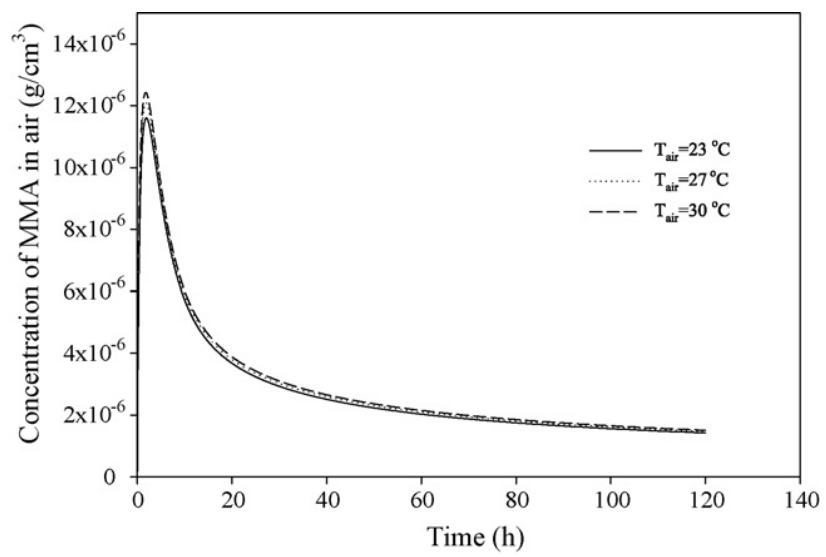

Fig. 9. The effect of air temperature on the concentration of MMA in air.

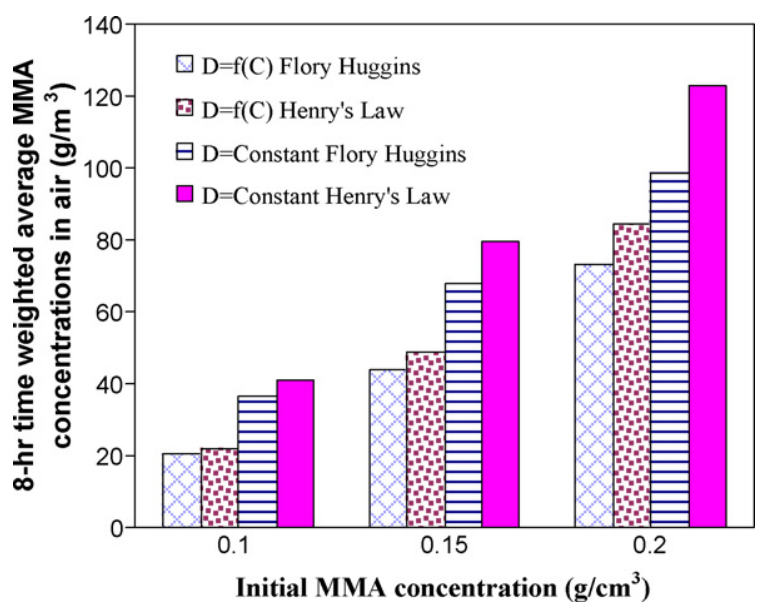

Fig. 10. 8-h time weighted average concentration of MMA in air predicted by utilizing different diffusivity and equilibrium relationships.

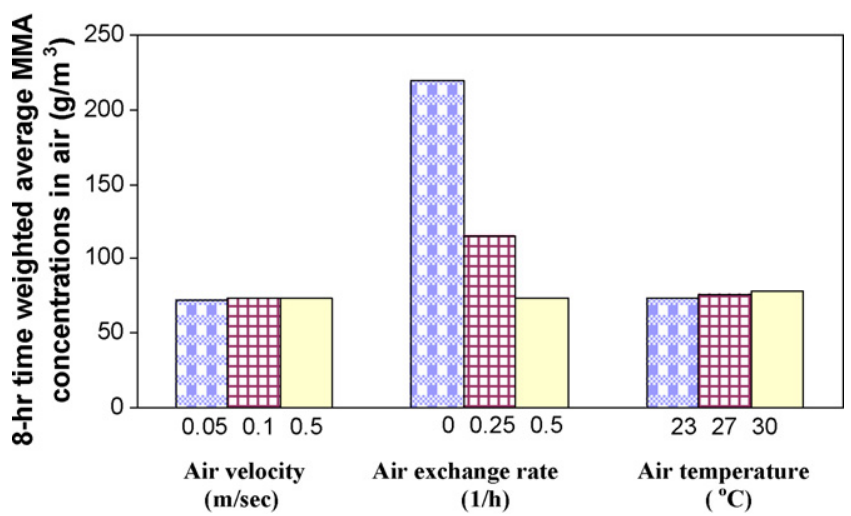

Fig. 11. The effects of the air velocity, air temperature and air exchange rate on 8-h time weighted average concentration of MMA in air.

concentrations of MMA are insensitive to both air velocity and temperature since at these levels of temperature and airflow, the rate of emission is mainly controlled by the diffusion of MMA in the coating rather than the external conditions. On the other hand, the results in Fig. 11 indicate that MMA levels in indoor air can be reduced dramatically by exchanging the air more frequently. As expected, the average MMA concentration in air increases proportionally with increased initial concentration in the coating, however, the coating thickness does not have a significant influence on the $8 \mathrm{~h}$

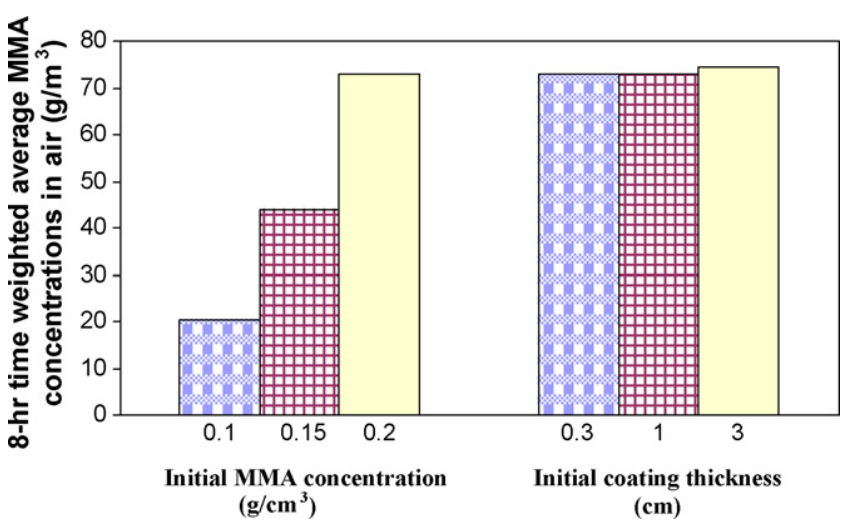

Fig. 12. The effects of the initial concentration of MMA and the initial coating thickness on 8-h time weighted average concentration of MMA in air. 
time weighted average concentration of MMA as demonstrated in Fig. 12.

\section{Conclusion}

A mathematical model has been developed to predict the rate of VOC emission from wet coating materials and applied to MMA/acrylic coating system. The model takes into account both internal and external mass transfer resistances along with possible moving boundary effects due to emission. Two distinct features of the model are concentration dependent VOC diffusivities in the coating and nonlinear equilibrium relationship which were described by Vrentas-Duda free volume theory and Flory Huggins theory, respectively.

The predictions have shown that the diffusivity of MMA decreases by almost two orders of magnitude as initially wet coating becomes dry. In addition, significant deviation occurs from linear equilibrium isotherm during the initial stages of emission when the MMA concentration in the coating is high. By utilizing constant diffusivity approximation or linear equilibrium isotherm or both, the emission rate of MMA from the coating, hence, MMA levels in air are predicted higher. The results suggest that such approximations are not valid for wet coating materials with the glass transition temperatures close to or higher than the room temperature and may lead to a wrong conclusion about the total emission time of the VOCs or wrong decisions on the design of ventilation system, etc. The emission of MMA is dominantly controlled by internal diffusion especially at later stages since the diffusivity of MMA within the coating becomes very small.

The model establishes a framework to quantitatively evaluate the effects of room conditions such as air velocity, air exchange rate and temperature on the emission rates. It can also be utilized to test the emission characteristics of various coating materials once the free volume parameters required to predict diffusivity and Flory Huggins interaction parameter of VOCs in the coating material are known. Such an approach could significantly reduce the need of measurements in environmental chambers which are usually expensive and time-consuming. The main intent of this study is to discuss the validity of the constant diffusivity and linear equilibrium relationship approximations so that their practical utility can be reconsidered by the research community. Further studies are certainly needed to evaluate the predictive ability of the proposed model through VOC measurements collected in real indoor environment or in small environmental chambers.

\section{References}

[1] Y.M. Kim, S. Harrad, R.M. Harrison, Concentrations and sources of VOCs in urban domestic and public microenvironments, Environmental Science and Technology 35 (2001) 997-1004.

[2] J.E. Dunn, Models and statistical methods for gaseous emission testing of finite sources in well-mixed chambers, Atmospheric Environment 21 (1987) 425-430.

[3] T.G. Matthews, A.R. Hawthorne, C.V. Thompson, Formaldehyde sorption and desorption characteristics of gypsum wallboard, Environmental Science and Technology 21 (1987) 629-634.

[4] J.C.S. Chang, Z. Guo, Characterization of organic emissions from a wood finishing product-wood stain, Indoor Air 2 (1992) 146-153.

[5] P.A. Clausen, Emission of volatile and semivolatile organic compounds from waterborne paints-the effect of the film thickness, Indoor Air 3 (1993) 269-275.

[6] L.E. Sparks, Z. Guo, J.C. Chang, B.A. Tichenor, Volatile organic compound emissions from latex paint. Part 1. Chamber experiments and source model development, Indoor Air 9 (1999) 10-17.

[7] J.W. Axley, Adsorption modeling for building contaminant dispersal analysis, Indoor Air 2 (1991) 147-171.

[8] B.A. Tichenor, Z. Guo, L.E. Sparks, Fundamental mass transfer model for indoor air emissions from surface coatings, Indoor Air 3 (1993) 263-268.
[9] Z. Guo, L.E. Sparks, B.A. Tichenor, J.C.S. Chang, Predicting the emissions of individual VOCs from petroleum based indoor coatings, Atmospheric Environment 32 (1998) 231-237.

[10] L.E. Sparks, B.A. Tichenor, J. Chang, Z. Guo, Gas-phase mass transfer model for predicting volatile organic compound (VOC) emission rates from indoor pollutant sources, Indoor Air-International Journal of Indoor Air Quality and Climate 6 (1996) 31-40.

[11] J.C. Little, A.T. Hodgson, A.J. Gadgil, Modeling emissions of volatile organic compounds from new carpets, Atmospheric Environment 28 (1994) 227-234.

[12] D. Kumar, J.C. Little, Characteristic of the source/sink behavior of double-layer building materials, Atmospheric Environment 37 (2003) 5529-5537.

[13] X. Yang, Q. Chen, J.Zeng, J.S. Zhang, C.Y. Shaw, A mass transfer model for simulating volatile organic compound emissions from 'wet' coating materials applied to absorptive substrates, International Journal of Heat and Mass Transfer 44 (2001) 1803-1815.

[14] X. Yang, Q. Chen, J.S. Zhang, R. Magee, J. Zeng, C.Y. Shaw, Numerical simulation of VOC emissions from dry materials, Building and Environment 36 (2001) 1099-1107.

[15] X. Yang, Q. Chen, J. Zeng, J.S. Zhang, C.Y. Shaw, Effects of environmental and test conditions on voc emissions from "Wet" coating materials, Indoor Air 11 (2001) 270-278.

[16] H. Huang, F. Haghighat, Modelling of volatile organic compounds emission from dry building materials, Building and Environment 37 (2002) 1127-1138.

[17] H.Y. Huang, F. Haghighat, Modeling of volatile organic compounds emission from dry building materials, Building and Environment 37 (2002) 1349-1360.

[18] T. Cheng, Y. Jiang, Y. Xu, Y. Zhang, Mathematical model for simulation of VOC emissions and concentrations in buildings, Atmospheric Environment 36 (2002) 5025-5030.

[19] F. Haghighat, H. Huang, Integrated IAQ model for prediction of VOC emissions from building material, Building and Environment 38 (2003) 1007-1017.

[20] Y. Xu, Y.P. Zhang, An improved mass transfer based model for analyzing VOC emissions from building materials, Atmospheric Environment 37 (2003) 2497-2505.

[21] B.Q. Deng, N.C. Kim, An analytical model for VOC emission from dry building materials, Atmospheric Environment 38 (2004) 1173-1180.

[22] X. Yang, J. Srebric, X. Li, G. He, Performance of three air distribution systems in VOC removal from an area source, Building and Environment 39 (2004) 1289-1299.

[23] Y. Xu, Y. Zhang, A general model for analyzing single surface VOC emission characteristics from building materials and its application, Atmospheric Environment 38 (2004) 113-119.

[24] C.S. Lee, F. Haghighat, W.S. Ghaly, A study on VOC source and sink behavio in porous building materials-analytical model development and assessment Indoor Air 15 (2005) 183-196.

[25] F. Li, J. Niu, L. Zhang, A physically-based model for prediction of VOCs emissions from paint applied to an absorptive substrate, Building and Environment 41 (2006) 1317-1325.

[26] F. Li, J. Niu, Control of volatile organic compounds indoors-Development of an integrated mass-transfer-based model and its application, Atmospheric Environment 41 (2007) 2344-2354.

[27] H.P. Hu, Y.P. Zhang, X.K. Wang, J.C. Little, An analytical mass transfer model for predicting VOC emissions from multi-layered building materials with convective surfaces on both sides, International Journal of Heat and Mass Transfer 50 (2007) 2069-2077.

[28] W. Yan, Y. Zhang, X. Wang, Simulation of VOC emissions from building materials by using the state-space method, Building and Environment 44 (2009) 471-478.

[29] J.S. Vrentas, J.L. Duda, Diffusion in polymer solvent systems: re-examination of the free volume theory, Journal of Polymer Science Part B-Polymer Physics 15 (1977) 403-416.

[30] J.S. Vrentas, J.L. Duda, Diffusion in polymer solvent systems: ii a predictive theory for the dependence of diffusion coefficients on temperature concentration and molecular weight, Journal of Polymer Science Part B-Polymer Physics 15 (1977) 417-439.

[31] J.L. Duda, J.S. Vrentas, S.T. Ju, H.T. Liu, Prediction of diffusion coefficients for polymer solvent systems, AICHE Journal 28 (1982) 279-285.

[32] P.J. Flory, Principles of Polymer Chemistry, Cornell Univ. Press, Ithaca, NY, 1953

[33] J.S. Vrentas, C.M. Vrentas, I. Hadj Romdhane, Analysis of inverse gas chromatography experiments, Macromolecules 26 (1993) 6670-6672.

[34] S. Alsoy, J.L. Duda, Modeling of multicomponent drying of polymer films, AICHE Journal 45 (1999) 896-905.

[35] Y. Yurekli, S. Alsoy Altinkaya, J.M. Zielinski, Solubility and diffusivity of methylmethacrylate and butylacrylate monomers in a MMA-BA copolymer, Journal of Polymer Science: Part B: Polymer Physics 45 (2007) 1996-2006.

[36] F. Haghighat, Y. Zhang, Modelling of emission of volatile organic compounds from building materials-estimation of gas-phase mass transfer coefficient, Building and Environment 34 (1999) 377-389.

[37] T.E. Daubert, R.P. Danner, Physical and Thermodynamic Properties of Pure Chemicals: Data Compilation, Hemisphere Publishing, New York, 1989.

[38] A.L. Tiffonnet, P. Blondeau, F. Allard, F. Haghighat, Sorption isotherms of acetone on various building materials, Indoor and Built Environment 11 (2002) 95-104.

[39] J.S. Chiou, Y. Maeda, D.R. Paul, Gas and vapor sorption in polymers just below TG, Journal of Applied Polymer Science 30 (1985) 4019-4029. 
[40] Y. Maeda, D.R. Paul, Effect of antiplasticization on gas sorption and Transport 1 polysulfone, Journal of Polymer Science Part B-Polymer Physics 25 (1987) 957-980.

[41] Y. Maeda, D.R. Paul, Effect of antiplasticization on gas sorption and Transport 2 poly(phenylene oxide), Journal of Polymer Science Part B-Polymer Physics 25 (1987) 981-1003.

[42] W.R. Vieth, J.M. Howell, J.H. Hsieh, Dual sorption theory, Journal of Membrane Science 1 (1976) 177-220.
[43] J.M. Zielinski, J.L. Duda, Predicting polymer solvent diffusion-coefficients using free volume theory, AICHE Journal 38 (1992) 405-415.

[44] S.S. Cox, J.C. Little, A.T. Hodgson, Effect of glass transition temperature on volatile emissions from polymer materials, in: Indoor Air 2005: Proceedings of the 10th International Conference on Indoor Air Quality and Climate 1-5, 2005, pp. 1845-1849. 